\title{
SwitchSENSE: a new technology to study protein-RNA interactions
}

Antoine Cléry ${ }^{1 *}$, Thibault J.M. Sohier ${ }^{1 \#}$, Thomas Welte ${ }^{2}$, Andreas Langer ${ }^{2}$, and Frédéric H.T. Allain $^{1}$ *

${ }^{1}$ Institute of Molecular Biology and Biophysics, Department of Biology, ETH Zurich, CH-8093

Zurich, Switzerland

${ }^{2}$ Dynamic Biosensors GmbH, Lochhamer Str. 15, 82152 Martinsried, Germany

"Master Biosciences de l’ENS Lyon, 69007 Lyon, France

*Authors to whom correspondence should be addressed.

allain@mol.biol.ethz.ch

aclery@mol.biol.ethz.ch

\begin{abstract}
Characterization of RNA-binding protein interactions with RNA became inevitable to properly understand the cellular mechanisms involved in gene expression regulation. Structural investigations bring information at the atomic level on these interactions and complementary methods such as Isothermal Titration Calorimetry (ITC) and Surface Plasmon Resonance (SPR) are commonly used to quantify the affinity of these RNA-protein complexes and evaluate the effect of mutations affecting these interactions. The switchSENSE technology has recently been developed and already successfully used to investigate protein interactions with different types of binding partners (DNA, protein/peptide or even small molecules). In this study, we show that this method is also well suited to study RNA binding proteins (RBPs). We could successfully investigate the binding to RNA of three different RBPs (Fox-1, SRSF1 and Tra2- $\beta 1$ ) and obtained $\mathrm{K}_{\mathrm{D}}$ values very close to the ones determined previously by SPR or ITC for these complexes. These results show that the switchSENSE technology can be used as an alternative
\end{abstract}


method to study protein-RNA interactions with $\mathrm{K}_{\mathrm{D}}$ values in the low micromolar $\left(10^{-6}\right)$ to nanomolar $\left(10^{-7}\right.$ to $\left.10^{-9}\right)$ and probably picomolar $\left(10^{-10}\right.$ to $\left.10^{-12}\right)$ range. The absence of labelling requirement for the analyte molecules and the use of very low amounts of protein and RNA molecules make the switchSENSE approach very attractive compared to other methods. Finally, we discuss about the potential of this approach in obtaining more sophisticated information such as structural conformational changes upon RBP binding to RNA.

\section{Keywords}

Protein, RNA, interaction, $\mathrm{K}_{\mathrm{D}}$, kinetics, switchSENSE

\section{Introduction}

RNA is a very sophisticated molecule that in addition to be a required intermediate between DNA and proteins was shown to play multiple crucial roles in gene expression [1]. As a short molecule (e.g. miRNA, piRNA, siRNA, snoRNA or snRNA) it can form RNA-RNA duplexes and be involved in post-transcriptional regulation events (e.g. gene expression silencing or maturation of long RNA precursors). As a longer molecule, RNA can adopt different secondary and tertiary structures, have a catalytic activity (e.g. ribozyme) or recruit RNA binding proteins (RBPs) at the place where they have to act in cells. Finally, RNA molecules are often post-transcriptionally modified (e.g. pseudouridylation, 2'-O-methylation or base editing) adding another layer of complexity to the regulation of their activity [2].

Protein-RNA interactions are heavily used for the regulation of most of the major cellular mechanisms involved in gene expression (e.g. transcription regulation, post-transcriptional silencing or epigenetic regulation) [3] explaining why impairment of protein binding to their RNA target sequences often leads to mis-regulation of cellular pathways, which can contribute to human diseases [4]. The study of protein-RNA complexes is therefore required to better understand the regulation of gene expression and may lead to new therapeutic strategies. It is thus important to develop new technologies to dissect these interactions. Structure determination

of protein-RNA complexes is typically used to decipher at the atomic level the mode of action of 
RBPs [5]. To complement these structural studies, other methods like Isothermal Titration Calorimetry (ITC) or Surface Plasmon Resonance (SPR) are very often used to determine the impact of a mutation in RNA or protein molecules on the affinity of the complexes and identify the contacts that are the most important for their formation. Recently, a new technology called switchSENSE $^{\circledR}$ has been developed at Dynamic Biosensors (DBS) GmbH (Martinsried, Germany). It utilizes biochips on which the DNA or protein of interest can be immobilized. The DRX analyser allows the rapid determination of the binding affinity and kinetics $\left(\mathrm{K}_{\mathrm{D}}, \mathrm{k}_{\mathrm{on}}\right.$ and $\mathrm{k}_{\mathrm{off}}$ ) of protein-DNA, protein-protein and protein-small molecule interactions [6-8]. The principle of the switchSENSE technology is the controlled movement (switching) of short surface grafted DNA (DNA nanolevers) on gold microelectrodes, actuated by alternating electric potentials. Depending on the potential that is applied to the electrodes, the DNA is either repelled from or attracted to the gold surface. The resulting movement of the DNA molecules is monitored in real time by the emission of a fluorescent dye attached to the distal end of covalently immobilized DNA strands (anchor strands) [9]. A DNA strand complementary to the anchor strands can be functionalized with a ligand molecule which principally can be any molecule that allows conjugation with standard chemical and biochemical methods. Furthermore, the second strand itself can serve as the target in an interaction analysis experiment. This allows experiments to be performed with a large variety of nucleic acid constructs. For instance, in this work a single stranded RNA molecule is hybridized to the anchor strand and features a short single stranded overhang that serves as target for protein binding studies. As the switching of the DNA nanolevers occurs in aqueous environment (buffer), hydrodynamic friction is the decisive parameter that affects the switching speed. The covalent attachment of ligand molecules to the nanolevers will add additional hydrodynamic drag, resulting in a deceleration of the switching motion. Further deceleration will be caused by the binding of analyte molecules to the immobilized ligands. Such changes of the switching dynamics can be observed in real time and allow to characterize interactions by analysis of the binding kinetics [6,10]. In addition, the degree of deceleration of the nanolevers correlates with the size of the attached molecule (large molecules cause more hydrodynamic friction than small molecules). This effect is used to determine the hydrodynamic diameters of proteins attached to the nanolevers and also of ligand/analyte complexes [11]. 
In this manuscript, we show that switchSENSE is a very promising method to measure affinity and kinetics of RBPs and we present the advantages and limitations of this new approach in this context.

\section{Material and methods}

\subsection{Preparation of proteins and RNA}

Two hybridized complementary ssDNA containing the T7 promotor followed by a GAG motif, which facilitates transcription initiation, the sequence to be transcribed and a BsaI restriction site were cloned into the pUC19 vector. Linearization of the plasmid by the BsaI restriction enzyme was used to stop transcription at the extremity of the sequence of interest. RNA was then transcribed in vitro using the T7 RNA polymerase and purified by HPLC as described previously [12]. Fox-1 RRM F126H and S166C were generated by directed mutagenesis. The UGCAUGU RNA oligonucleotide was purchased from Dharmacon, deprotected according to the manufacturer's instructions, lyophilised and resuspended in T140 buffer.

RRM of Tra2- $\beta 1$ (106-201), RRM of WT and mutated versions of Fox-1 (109-208) and RRM2 of SRSF1 (107-203) containing N-terminal hexa-histidine tags (and an additional GB1 solubility tag in SRSF1 RRM2) were expressed in E. coli BL21 (DE3) codon plus strains at $37^{\circ} \mathrm{C}$ in LuriaBertani media. Proteins were purified by two successive nickel affinity chromatography (QIAGEN) steps using their N-terminal His tag. SRSF1 RRM2 was dialysed in T40 buffer (10mM Tris- $\mathrm{HCl} \mathrm{pH} 7.4,40 \mathrm{mM} \mathrm{NaCl}$ and $0.05 \%$ tween20). Tra2- $\beta 1$, Fox-1 WT and F126H RRMs were dialysed against T140 buffer (10mM Tris- $\mathrm{HCl} \mathrm{pH} \mathrm{7.4,} \mathrm{140mM} \mathrm{NaCl} \mathrm{and} 0.05 \%$ tween20). Both buffers are optimized for switchSENSE measurements. Fox-1 S166C RRM was dialysed in T40 buffer and then covalently attached to DNA complementary to the nanolevers using the thiol coupling kit provided by DBS.

\section{2 switchSENSE kinetics measurements}


All switchSENSE experiments were performed on a DRX analyser using MPC-48-2-Y1$\mathrm{S}$ chips (both Dynamic Biosensors GmbH, Martinsried, Germany). The ssDNA sequence attached to the chip (nanolever) was optimized by DBS for thermal stability, absence of secondary structures, hybridization efficiency and minimal cross-reactivity with reference strands. 30ul of RNA resuspended in water at a concentration of $1 \mu \mathrm{M}$ was needed to saturate the ssDNA molecules attached to electrodes. After completion of the hybridization process, excess RNA was washed away with running buffer. A regeneration solution provided by DBS (alkaline solution at $\mathrm{pH}$ 13) was used to remove RNA from the nanolever after each titration step. $30 \mu \mathrm{l}$ of Fox-1 S166C conjugate was used at a concentration of $200 \mathrm{nM}$ to saturate the ssDNA molecules attached to electrodes. After completion of the hybridization process, excess of Fox-1 S166CDNA conjugates was washed away with running buffer.

For Fox-1 RRM, $250 \mu 1$ of protein (at concentrations of 26.3, 39.5, 59.3, 88.9, 133 and $200 \mathrm{nM}$ ) was injected with a flow rate of $50 \mu \mathrm{l} / \mathrm{min}$ and all measurements were performed at $25^{\circ} \mathrm{C}$. For Tra2- $\beta 1 \mathrm{RRM}, 9 \mu 1$ of protein (at concentrations of 1, 1.6, 2.4, 3.6, 5.3 and $8 \mu \mathrm{M}$ ) was injected with a flow rate of $10 \mu \mathrm{l} / \mathrm{min}$ and all measurements were performed at $40^{\circ} \mathrm{C}$. For SRSF1 RRM2, $100 \mu$ l of protein (at concentrations of 188, 375, 750 and $1500 \mathrm{nM}$ ) was injected with a flow rate of $50 \mu \mathrm{l} / \mathrm{min}$ and all measurements were performed at $37^{\circ} \mathrm{C}$. For the three proteins, dissociation was measured with a flow rate of $50 \mu \mathrm{l} / \mathrm{min}$. The synthetic UGCAUGU RNA was injected with a flow rate of $100 \mu \mathrm{l} / \mathrm{min}$ at $25^{\circ} \mathrm{C}$ and dissociation was measured with a flow rate of $50 \mu \mathrm{l} / \mathrm{min}$.

Analysis was performed with the switchANALYSIS® software from Dynamic Biosensors. Normalized Dynamic Response values were obtained by subtracting the signal measured upon buffer injection from each titration measurements. The association and dissociation rate constants $\left(\mathrm{k}_{\mathrm{on}}\right.$ and $\left.\mathrm{k}_{\mathrm{off}}\right)$ and the respective error values were derived from a global single exponential fit model. Whenever several concentration steps were evaluated, the shown error reflects the overall error of the global fit for all measurements.

\subsection{Biochip compatibility with measurements of protein-RNA interactions}


In general, most buffer solutions that are used for standard biochemical and biophysical experiments are compatible with the switchSENSE technology. $\mathrm{pH}$ can range between 5 and 10. Measurements that are performed in fluorescence mode (in the absence of electrical actuation) are usually not affected by changes of the buffer conditions, if running buffer and sample buffer match. In principle, they could even be performed in the presence of up to $3 \mathrm{M}$ monovalent salts.

However, contrary to the fluorescence, the switching dynamics of DNA are affected by the salt concentration present in the measurement buffer. Notably, the signal to noise ratio is impaired at high salt concentrations. Therefore, concentrations of monovalent salts can be used at concentrations ranging from 1 to $300 \mathrm{mM}$ if electrical actuation (switching mode) of the DNA is required. Yet, this strongly depends on the individual experiment as various other parameters have an influence on the switching behavior. For example the size, shape and flexibility of both ligand and analyte and the length of the DNA nanolever have some influence on this. Thus, in some cases it can be beneficial to optimize the buffer compositions.

Other additives are in most cases unproblematic. For instance, detergents (DDM, Tween20, Sarcosyl) can be used without limitations at critical micelle concentration (CMC). Furthermore, reagents that bring along changes of the refractive index, for instance DMSO, are usually not interfering with the switchSENSE technology.

Finally, buffers containing reducing agents can require optimization. Thiol based reducing agents (DTT, $\beta$-mercaptoethanol) are critical in DNA switching experiments as they will disrupt the gold-sulfur bond that is used for DNA immobilization. Nevertheless, other reducing agents, such as TCEP, are generally compatible. In fluorescence measurement modes, all reducing agents are compatible at standard concentrations.

Importantly, each channel can be used several times. The number of possible chip regenerations depends strongly on the experimental conditions. Under the conditions that were used in this study, one flow channel was usually loaded with fresh RNA for about 50 times. Under harsh conditions, for instance when measuring at higher temperatures, 
this number can be decreased. Nevertheless, under optimum conditions more than 100 regenerations cycles of one flow channel can be achieved.

\section{Results}

\subsection{Transcription and hybridization of the RNA of interest on the biochip}

The switchSENSE instrument is particularly well suited to study RBPs since the RNA of interest can be transcribed and annealed to the single-stranded DNA (ssDNA) attached to the electrodes of the biochip. For this study, we used long RNA sequences (around 60nts) for which the 3'-extremity is fully complementary to the 48-nts long ssDNAs attached to the electrodes and the 5'-part corresponds to a flanking non-hybridized sequence containing a linker of 4 uridines, the motif bound by the protein and the GAG transcription start motif (Fig. 1A). RNAs were very efficiently transcribed in vitro using the T7 RNA polymerase and purified by HPLC. We then had to test whether this purified RNA could be efficiently hybridized to the complementary ssDNA, which is covalently attached to the electrodes. RNA hybridization was investigated by following the increase of fluorescence upon formation of RNA-DNA duplexes (Fig. 1B). This was based on the observation that the duplex is repulsed more efficiently from the gold surface than non-hybridized ssDNA when a negative potential is applied to the electrodes (Fig. 1B). We tested different RNA concentrations and observed an efficient and reproducible hybridization to the ssDNA using a final RNA concentration of $1 \mu \mathrm{M}$ in water or T40 buffer (Fig. 1B). We also noticed that increasing the temperature of hybridization to $37^{\circ} \mathrm{C}$ was improving the ssDNA-RNA duplex formation, most likely by limiting the formation of RNA secondary structures. We also checked that the hybridized RNA could be completely removed from the electrodes after a regeneration step using the regeneration buffer provided by Dynamic Biosensors. This was needed to have the possibility to charge the electrodes with a new RNA molecule after each titration or kinetic step.

\subsection{Setup of switchSENSE kinetics measurements}


We then tested whether the binding of proteins to the flanking ssRNA sequence of interest could be detected by the switchSENSE technology (Fig. 1). We decided to work with RNA Recognition Motifs (RRMs), because they interact with ssRNA motifs using a broad range of affinities ( $\mathrm{K}_{\mathrm{D}}$ values from low micromolar to nanomolar range) [5]. We started with the Fox-1 RRM, which was shown by SPR to bind to 5'-UGCAUGU-3' RNA with a $K_{D}$ of $1.09 \mathrm{nM}$ [13]. Using this information, we transcribed and purified an RNA molecule containing this sequence in the 5' non-hybridized part (Fig. 1A) and predicted the experimental conditions using the switchBUILD software provided by Dynamic Biosensors. When no information is available on the $\mathrm{K}_{\mathrm{D}}$ of the protein-RNA complex of interest, the user will first need to gradually increase the protein concentrations until he observes a change of the fluorescence or dynamic response signal. The estimated $K_{D}$ value is then usually around 10-fold higher than the minimal concentration value required to observe a binding of the protein to the RNA target. Using this estimated $K_{D}$ value, switchBUILD automatically calculates the protein concentrations and volumes that should be used for the kinetics and adapts the flow rates and durations of association and dissociation steps. Typically, the software proposes a concentration regime for the analyte that would yield significant signal changes within five minutes of association time. The respective dissociation time is proposed based on the dissociation rate constant $\left(\mathrm{k}_{\mathrm{off}}\right)$ as multiples of the dissociation time constant. Furthermore, switchBUILD provides the possibility to adjust all experimental parameters manually. The script generated with this software can then be opened directly in the switchCONTROL software, which controls the machine. For kinetics measurements, we recommend to remove and hybridize fresh RNA molecules after each dissociation step to be sure that all bound proteins are completely removed from the electrodes before starting a new protein association step. The association of the protein to the RNA of interest and the following dissociation is typically characterized by using different protein concentrations to obtain accurate and reproducible $\mathrm{k}_{\mathrm{on}}, \mathrm{k}_{\mathrm{off}}$ and $\mathrm{K}_{\mathrm{D}}$ values averaged on these independent experiments.

\section{3 switchSENSE kinetics measurements in the presence of RBPs}


One of the advantages of the switchSENSE approach consists of the possibility to obtain kinetics parameters using two independent methods. A fluorescent molecule is present at the 3'extremity of the ssDNA attached to the electrode (Fig. 1A). The signal can either decrease by quenching or be enhanced upon binding of the protein to the hybridized RNA molecule and be followed during association and dissociation of the protein. This method is particularly suited to the study of interactions between two biomolecules of very different sizes (e.g. protein interactions with small molecules). In parallel, it is also possible to follow the switching speed of the DNA-RNA duplex upon protein association and dissociation (Fig. 1C). Binding of the protein to RNA decreases this speed due to increased hydrodynamic friction, whereas the dissociation has the opposite effect (Fig. 1D). To characterize the DNA switching speed, a dynamic response (DR) value is constantly extracted from the observation of the DNA motion (Fig. 1C) and followed upon time (Fig. 1D). Whereas the observed signal changes in fluorescence were not high enough to determine kinetics parameters for the three protein-RNA complexes tested in this study, we obtained very reproducible $K_{D}$ values by following the switching speed of the DNA-RNA duplexes upon protein association and dissociation. Therefore, these two approaches seem to be complementary depending on the type of interactions that need to be studied.

In this study, we decided to focus on three protein-RNA complexes based on their differences in affinities for RNA: Fox-1 RRM $\left(K_{D}=1.09 \mathrm{nM}\right)$ [13], SRSF1 RRM2 $\left(\mathrm{K}_{\mathrm{D}}=0.8 \mu \mathrm{M}\right)$ [14] and Tra2- $\beta 1$ RRM $\left(K_{D}=2.25 \mu M\right)$ [15]. The affinity of Fox-1 RRM has previously been determined by SPR and we first tested whether it would be possible to measure a similar $K_{D}$ value with the switchSENSE method. We used 6 different protein concentrations and could well describe the data using a global single-exponential fit (Fig. 2A). We obtained a $\mathrm{K}_{\mathrm{D}}$ of $1.49 \mathrm{nM}$, in good agreement with the SPR data, whereas the $\mathrm{k}_{\text {on }}$ and $\mathrm{k}_{\text {off }}$ values were different. However, a direct comparison of the rate constants is complicated by the fact that different fit models were used for the data analysis: the SPR data did not follow a simple exponential time course, but had to be fitted with a complex mass-transport limited fit-model including offsets accounting for incomplete dissociation, whereas switchSENSE data could be described well by simple singleexponential fits as expected for a 1:1 interaction. We also verified that this interaction was specific by investigating the binding of Fox-1 RRM with a polyU sequence. As illustrated in Figure 2B, no interaction could be detected with all protein concentrations tested. 
We then tested whether switchSENSE could be used to detect small differences in affinity between WT Fox-1 RRM and proteins for which residues involved in RNA binding had been mutated. We measured some kinetics with the F126H mutated version of Fox-1 RRM, which was also previously measured by SPR ( $\mathrm{K}_{\mathrm{D}}$ of $\left.22.1 \mathrm{nM}\right)$ [13] and obtained a very similar $\mathrm{K}_{\mathrm{D}}$ value (25.3 nM) (Fig. 2C). In another study we could even detect reproducibly differences in affinity between WT and mutated Fox-1 proteins with smaller difference of 2 to 5-fold (with Fox-1 S122A and S155A, respectively) (Krepl et al., in preparation). Finally, we tested the possibility to attach directly Fox-1 RRM to the biochip using a kit provided by DBS, which allows the covalent ligation of cysteines to ssDNA molecules complementary to the nanolevers. As no cysteine was present in the sequence of Fox-1 RRM, we converted serine 166, which is not involved in RNA binding and is solvent accessible (located in the $\beta 3-\alpha 2$ loop), into a cysteine residue [13]. In this context, we could not detect a significant modification of the dynamic response when the immobilized conjugate was incubated with RNA. However, the fluorescence signal was clearly increasing upon RNA binding (Fig. 2D) and we could determine a $K_{D}$ value very close to the one obtained in Fig. $2 \mathrm{~A}$ ( $\mathrm{K}_{\mathrm{D}}$ values of $4.39 \mathrm{nM}$ and $1.49 \mathrm{nM}$, respectively). Nevertheless, we observed slightly different values of $\mathrm{k}_{\text {on }}$ and $\mathrm{k}_{\text {off }}$ between the two experiments that seems to originate from the attachment of the protein to the biochip.

We then tested whether switchSENSE was adapted for the determination of higher $K_{D}$ values. We investigated the interaction of another protein-RNA complex involving SRSF1 RRM2, a domain that was shown to interact with a 5'-GGA-3' RNA motif using a non-canonical mode of recognition and for which a $K_{D}$ of $0.8 \mu \mathrm{M}$ was determined by ITC in the presence of the 5'-UGAAGGAC-3' RNA [14]. Here also we used several protein concentrations and obtained a good global fit of the data over all concentrations tested. The $K_{D}$ value that we obtained was slightly lower than expected $(0.39 \mu \mathrm{M})$ (Fig. 3A). However, the buffer conditions were slightly different as we worked at $40 \mathrm{mM} \mathrm{NaCl}$ instead of the $100 \mathrm{mM} \mathrm{Arg} / \mathrm{Glu}$ buffer [14]. Similarly to Fox-1 RRM, no interaction was observed with the RNA used as a negative control (Fig. 3B).

Finally, we investigated whether protein-RNA interactions in the low micromolar range could also be investigated using the switchSENSE approach. We focused on Tra2- $\beta 1$ RRM for which we previously solved the structure bound to the 5'-AAGAAC-3' RNA and determined a $\mathrm{K}_{\mathrm{D}}$ value of $2.25 \mu \mathrm{M}$ using ITC [15]. As illustrated in Figure 4A, even in this range of affinities 
we could obtain global fits using 6 different protein concentrations and a $\mathrm{K}_{\mathrm{D}}$ of $2.89 \mu \mathrm{M}$ was derived from these data. No interaction was observed at these concentrations with a polyU RNA sequence (Fig. 4B). However, the protein started to interact non-specifically with this RNA at higher concentrations. Finally, we tested another approach to determine the $K_{D}$ value of this complex by reporting for different protein concentrations the DR values measured at the end of each association step. This titration experiment gives a plot that can be fitted using the Langmuir equation. We obtained a $K_{D}$ value of $1.53 \mu \mathrm{M}$ (Fig. 4C) close to the one determined by kinetics analysis (Fig. 4A).

\section{Discussion}

In this manuscript, we showed that the switchSENSE approach allows the determination of $\mathrm{k}_{\mathrm{on}}$ and $\mathrm{k}_{\text {off }}$ parameters for protein-RNA complexes with $\mathrm{K}_{\mathrm{D}}$ values in the low micromolar $\left(10^{-6}\right)$ to nanomolar $\left(10^{-7}\right.$ to $\left.10^{-9}\right)$ regime. These values were very close to the ones determined by SPR (Fox-1 RRM) [13] and ITC (SRSF1 RRM2 and Tra2- $\beta 1$ RRM) [14,15] measurements demonstrating that the switchSENSE technology can be used as an alternative method to study protein-RNA interactions.

\subsection{Advantages and limitations of the switchSENSE method}

The possibility to observe protein-RNA association and dissociation by following the switching speed or the variation in fluorescence intensity allows the investigation of very different types of interactions involving proteins, nucleic acids or small molecules. We observed that the analysis of the Dynamic Response signals seemed to be the most appropriate approach to follow protein-RNA kinetics when the RNA is attached to the biochip. Indeed, variations in the fluorescence intensity were too small to derive accurate $\mathrm{K}_{\mathrm{D}}$ values on the three complexes tested. Nevertheless, it was possible to determine $\mathrm{k}_{\text {on }}$ and $\mathrm{k}_{\text {off }}$ parameters in the fluorescence mode when the protein conjugate was hybridized to the nanolever instead of the RNA molecule (fig. 2D).

Contrary to other methods using fluorescence to detect protein-RNA interactions, the switchSENSE approach does not require any labeling of the biomolecules, because the 
fluorophore is already present at the 3' extremity of the ssDNA covalently attached to the biochip (fig. 1A). In addition, it requires smaller amount of material (Table 1) and is less expensive than other methods like ITC, which requires the use of costly small synthesized RNA when the protein of interest interacts with a short RNA sequence. Indeed, RNAs shorter than 20nts are very difficult to transcribe efficiently in vitro. The switchSENSE technology offers an interesting alternative to this issue as we showed in this manuscript that long RNAs can be transcribed in vitro and then hybridized to the ssDNA attached to the biochip with a flanking 5, extremity containing the ssRNA sequence of interest (Fig. 1A). Importantly, this strategy facilitates the production and use of RNA mutants. Alternatively, chimeric DNA-RNA molecules could be synthesized with the 3' DNA part complementary to the ssDNA attached to the electrodes and the RNA corresponding to the 5' flanking sequence of interest. In addition, it is also possible to immobilize the protein on the switchSENSE sensor electrode and then test the binding of the RNA molecule of interest (fig. 2D). Dynamic Biosensors provides multiple kits allowing a covalent attachment of the protein at the 5' extremity of a ssDNA molecule that is complementary to the ssDNA bound to the biochip. Also in contrast to ITC, which becomes less sensitive for the measurement of sub-nanomolar $K_{D}$, switchSENSE allows the determination of very low $K_{D}$ values as shown in this manuscript with Fox-1 RRM ( $K_{D}$ value of $\left.1.49 \mathrm{nM}\right)$ (Fig. 2A). Although we did not test protein-RNA interactions in the picomolar range, we believe that such investigation should even be possible as it was previously done using this technology for interactions between antigen and antibodies [8] and DNA polymerases [6].

Although this method is compatible with a large range of buffers, we noticed that some buffer optimization was required before obtaining the signal to noise expected in order to measure reliable $K_{D}$ values, which could be a limitation for some protein-RNA complexes requiring a very specific buffer. In addition, we noted that the analysis of the raw data of complex interactions is fairly delicate and requires experience and expertise. Finally, we observed the apparition of nonspecific interactions at Tra2- $\beta 1$ RRM concentrations above $8 \mu \mathrm{M}$, which may be a limitation to measure protein-RNA interactions with low affinities. Yet, it is not unusual for RNA or DNA binding proteins to observe a certain background affinity to nucleic acids in general. This effect is often owed to the positive charge of the protein $[16,17]$. Furthermore, interactions between proteins and nucleic acids are sometimes highly dependent on the buffer conditions. Notably the salt concentration, the presence of specific ions or of 
unspecific competitors can have a strong effect on the binding strength and specificity, thus providing the possibility to overcome issues linked to unspecific interaction of proteins with nucleic acids $[18,19]$. Alternatively, for protein interacting non-specifically with double-stranded nucleic acid molecules, a shorter DNA-RNA duplex could be tested.

\subsection{Potential of this approach for protein-RNA interactions}

In this study, we focused on protein-ssRNA interactions. Nevertheless, it should also be possible to work with dsRNA molecules by hybridizing a complementary RNA to the RNA overhang. A similar approach could be used to study protein binding to stem-loop RNA molecules using RNA with an elongated 3'-extremity complementary to the nanolever. Importantly, in addition to measure kinetics of protein-RNA interactions, the switchSENSE technology could be used for applications that were previously tested in the context of proteinDNA interactions and could possibly be transferred to protein-RNA complexes.

It was for example technically possible to follow protein-induced conformational changes in dsDNA as illustrated by the study published by Spuhler and co-workers that demonstrates a bending of the dsDNA molecule upon binding of the Escherichia coli integration host factor [20]. Even more impressive was the possibility to follow simultaneously and in real-time the binding of DNA polymerases to dsDNA and the subsequent DNA elongation [7]. It allowed the authors to observe changes in the DNA polymerase conformation (finger closing) during enzymatic activity [7]. Finally, the size of proteins bound to the biochip via their attachment to ssDNA complementary to the ssDNA bound to the electrodes can be estimated by switching the proteins over nanometer amplitudes and comparing their motional dynamics to a theoretical model [6]. This strategy could be used for example to follow dimerization or multimerisation of RBPs on RNA. The possibility to obtain information on size and conformational changes of the binding partners makes the switchSENSE technology very attractive and opens up the access to information on protein-RNA interaction that was not be accessible before.

\section{Conclusions}


We used for the first time the switchSENSE approach to study protein-RNA interactions. Although this method still needs to be challenged for protein-RNA complexes interacting with $\mathrm{K}_{\mathrm{D}}$ values higher than $2 \mu \mathrm{M}$, we showed that it was possible to quantify affinities in the low micromolar to nanomolar range. Protein and RNA partners do not need to be labeled and RNA can be produced by in vitro transcription, which facilitates the production of material required for this type of study. Following the dynamic response signal seems to be the most appropriate method to follow protein-RNA kinetics.

\section{Acknowledgements}

We thank Wolfgang Kaiser and Ulrich Rant from the Biosensor Dynamics Company for their comments on the manuscript.

\section{Conflict of interest}

All the experiments were performed in the laboratory of Prof. Frederic Allain using protein and RNA molecules produced in his lab and exclusively by persons that are not hired by Dynamic Biosensors GmbH. Thomas Welte and Andreas Langer, who worked in the Biosensor Dynamics Company helped us to setup the conditions and analyse the data in an appropriate manner.

\section{Funding}

FA and AC acknowledge support from the NCCR RNA and Disease funded by the Swiss National Science Foundation. AC acknowledges support from cure SMA and the Fondation Suisse de Recherche sur les Maladies Musculaires (FSRMM).

\section{Legends}

Figure 1: Description of the switchSENSE technology applied to protein-RNA interactions 
(A) Schematic view of RNA hybridization to the ssDNA covalently attached to the biochip electrodes. The sequence of ssDNA and RNA are shown. (B) Duplex DNA is repulsed more efficiently from the gold surface than non-hybridized ssDNA when a negative potential is applied to the electrodes. RNA hybridization is followed by measuring the increase of fluorescence upon time. (C) Application of an alternating voltage to the electrode induces a switching of the RNA-DNA duplex that becomes slower upon protein binding. This decrease in switching speed can be quantified by determination of the dynamic response (DR), which is derived from the time resolved fluorescence intensity changes, typically of the upward motion of the DNA. (D) Following the evolution of the DR signal allows the measurement of $k_{\text {on }}$ and $k_{\text {off }}$ parameters, which can then be used to calculate the $K_{D}$ value of protein-RNA complexes. Schemes shown in panels B to D were reprinted with permission from Dynamic Biosensors $\mathrm{GmbH}$.

Figure2: switchSENSE molecular dynamics measurements performed in the presence of Fox-1 RRM

Kinetic analysis of Fox-1 RRM WT (at concentrations of 26.3, 39.5, 59.3, 88.9, 133 and 200nM) interacting with the 5'- UGCAUGU-3' (A) and polyU (B) RNA sequences using the switchSENSE technology. (C) Kinetic analysis of Fox-1 RRM F126H (at concentrations of 26.3, 39.5, 59.3, 88.9, 133 and 200nM) interacting with the 5'- UGCAUGU-3' RNA sequence using the switchSENSE technology. (D) Kinetic analysis of Fox-1 RRM S166C attached to the biochip with increasing concentrations of 5'- UGCAUGU-3' RNA (at concentrations of 1, 2, 4 and 8 $n M)$. The raw data is superimposed by global exponential fits. $k_{\text {on }}, k_{\text {off }}$ and $K_{D}$ values are shown for each protein-RNA kinetics measurement.

Figure 3: SwitchSENSE molecular dynamics measurements performed in the presence of SRSF1 RRM2

Kinetic analysis of SRSF1 RRM2 WT (at concentrations of 188, 375, 750 and 1500nM) interacting with the 5'-UGAAGGAC-3' (A) and polyC (B) RNA sequences using the 
switchSENSE technology. The raw data is superimposed by global exponential fits. $\mathrm{k}_{\mathrm{on}}, \mathrm{k}_{\mathrm{off}}$ and $\mathrm{K}_{\mathrm{D}}$ values are shown for each protein-RNA kinetics measurement.

Figure 4: SwitchSENSE molecular dynamics measurements performed in the presence of

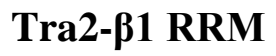

Kinetic analysis of Tra2- $\beta 1$ RRM WT (at concentrations of 1, 1.6, 2.4, 3.6, 5.3 and $8 \mu \mathrm{M}$ ) interacting with the 5'-AAGAAC-3' (A) and polyU (B) RNA sequences using the switchSENSE technology. The raw data is superimposed by global exponential fits. $\mathrm{k}_{\mathrm{on}}, \mathrm{k}_{\mathrm{off}}$ and $\mathrm{K}_{\mathrm{D}}$ values are shown for each protein-RNA kinetics measurement. (C) Titration analysis of Tra2- $\beta 1$ RRM WT (at concentrations of 5.24, 13.1, 32.8, 81.9, 205, 512, 1280, 3200 and 8000nM) interacting with the 5'-AAGAAC-3' RNA sequences using the switchSENSE technology. The data were analyzed using a 1:1 Langmuir fit. 


\begin{tabular}{|l|l|l|l|}
\hline & SPR (Biacore T200) & PEAQ-ITC & switchSENSE \\
\hline
\end{tabular}

Table1: comparison of three methods used to determine affinities of protein-RNA complexes. 


\begin{tabular}{|c|c|c|c|}
\hline $\begin{array}{l}\text { Duration of the } \\
\text { experiment }\end{array}$ & $\begin{array}{l}30 \text { min to several hours } \\
\text { depending on } k_{\text {on }} \text { and } k_{\text {off }} \\
\text { values }\end{array}$ & $\begin{array}{l}45 \text { min to several hours } \\
\text { depending on } K_{D} \text { values }\end{array}$ & $\begin{array}{l}30 \text { min to several hours } \\
\text { depending on } k_{\text {on }} \text { and } k_{\text {off }} \\
\text { values }\end{array}$ \\
\hline $\begin{array}{l}\text { Quantity of } \\
\text { RNA / } \\
\text { experiment }\end{array}$ & 1.5 pmol* & $10-15 \mathrm{nmol}$ & $3.0 \mathrm{pmol}^{* *}$ \\
\hline $\begin{array}{l}\text { Quantity of } \\
\text { protein / } \\
\text { experiment }\end{array}$ & $0.14 \mathrm{nmol}^{* * *}$ & $10-15 \mathrm{nmol}$ & $0.14 \mathrm{nmol}^{* * *}$ \\
\hline $\begin{array}{l}\text { General } \\
\text { features }\end{array}$ & $\begin{array}{l}\text { Surface based [21] } \\
\text { Biotinylation of RNA is } \\
\text { required for ligand } \\
\text { immobilization [13] } \\
\text { Measurement of affinity } \\
\text { and time-resolved kinetic } \\
\text { measurements [21] } \\
\text { Affinity range: fM-mM } \\
\text { (manufacturer's data) }\end{array}$ & $\begin{array}{l}\text { Solution based [21] } \\
\text { No immobilization required } \\
{[21]} \\
\text { Measurement of affinity and } \\
\text { thermodynamic parameters } \\
\text { [21] } \\
\text { Requires enthalpic changes for } \\
\text { detection [21] } \\
\text { Affinity range: } 10^{-12}-10^{-2} \mathrm{M} \\
\text { (manufacturer's data) }\end{array}$ & $\begin{array}{l}\text { Surface based [22] } \\
\text { Direct immobilization of } \\
\text { synthetic or natural RNA via } \\
\text { specific base pairing to } \\
\text { immobilized DNA nanolevers } \\
\text { (this work) } \\
\text { Regeneration process allows } \\
\text { measurements with unused } \\
\text { ligands that have not been } \\
\text { exposed to potentially } \\
\text { harmful conditions [10] } \\
\text { Measurement of affinity and } \\
\text { time time-resolved kinetic } \\
\text { measurements [10] } \\
\text { Affinity range: } 50 \mathrm{fM} \mathrm{-} 1 \mathrm{mM} \\
\text { (manufacturer's data) }\end{array}$ \\
\hline
\end{tabular}

* RNA immobilization conditions: $5 \mathrm{nM}$ biotinylated RNA; pump rate: $100 \mu \mathrm{L} / \mathrm{min}$; contact time: $180 \mathrm{~s}$. These conditions would typically yield full surface coverage at an association rate constant of $k_{\text {on }}=1.0 \times$ $10^{7} \mathrm{M}^{-1} \mathrm{~s}^{-1}$.The presented value is sufficient for one ligand immobilization step.

** RNA immobilization conditions: 100 nM RNA; stopped flow; volume: $30 \mu$. The presented value is sufficient for one ligand immobilization step. 
*** Sample consumption at the example of a kinetic experiment: Six increasing concentrations $(0.263$ $\mathrm{nM}-200 \mathrm{nM}$; dilution factor: 1.5); pump rate: $50 \mu \mathrm{l} / \mathrm{min}$; contact time: $300 \mathrm{~s}$.

\section{References}

1. Morris KV, Mattick JS: The rise of regulatory rna. Nat Rev Genet (2014) 15(6):423-437.

2. Gilbert WV, Bell TA, Schaening C: Messenger rna modifications: Form, distribution, and function. Science (2016) 352(6292):1408-1412.

3. Re A, Joshi T, Kulberkyte E, Morris Q, Workman CT: Rna-protein interactions: An overview. Methods Mol Biol (2014) 1097(491-521.

4. Lukong KE, Chang KW, Khandjian EW, Richard S: Rna-binding proteins in human genetic disease. Trends Genet (2008) 24(8):416-425.

5. Afroz T, Cienikova Z, Clery A, Allain FH: One, two, three, four! How multiple rrms read the genome sequence. Methods Enzymol (2015) 558(235-278.

6. Langer A, Hampel PA, Kaiser W, Knezevic J, Welte T, Villa V, Maruyama M, Svejda M, Jahner S, Fischer $\mathrm{F}$, Strasser $\mathrm{R}$ et al: Protein analysis by time-resolved measurements with an electroswitchable DNA chip. Nat Commun (2013) 4(2099.

7. Langer A, Schraml M, Strasser R, Daub H, Myers T, Heindl D, Rant U: Polymerase/DNA interactions and enzymatic activity: Multi-parameter analysis with electro-switchable biosurfaces. Sci Rep (2015) 5(12066.

8. Lux G, Langer A, Pschenitza M, Karsunke X, Strasser R, Niessner R, Knopp D, Rant U: Detection of the carcinogenic water pollutant benzo[a]pyrene with an electro-switchable biosurface. Anal Chem (2015) 87(8):4538-4545.

9. Rant U, Arinaga K, Fujita S, Yokoyama N, Abstreiter G, Tornow M: Dynamic electrical switching of DNA layers on a metal surface. Nano Letters (2004) 4(12):2441-2445.

10. Knezevic J, Langer A, Hampel PA, Kaiser W, Strasser R, Rant U: Quantitation of affinity, avidity, and binding kinetics of protein analytes with a dynamically switchable biosurface. $\mathrm{J} \mathrm{Am} \mathrm{Chem}$ Soc (2012) 134(37):15225-15228. 
11. Langer A, Kaiser W, Svejda M, Schwertler P, Rant U: Molecular dynamics of DNA-protein conjugates on electrified surfaces: Solutions to the drift-diffusion equation. $J$ Phys Chem $B$ (2014) 118(2):597-607.

12. Dominguez $\mathrm{C}$, Schubert M, Duss O, Ravindranathan S, Allain FH: Structure determination and dynamics of protein-rna complexes by nmr spectroscopy. Prog Nucl Magn Reson Spectrosc (2011) 58(1-2):1-61.

13. Auweter SD, Fasan R, Reymond L, Underwood JG, Black DL, Pitsch S, Allain FH: Molecular basis of rna recognition by the human alternative splicing factor fox-1. EMBO J (2006) 25(1):163-173.

14. Clery A, Sinha R, Anczukow O, Corrionero A, Moursy A, Daubner GM, Valcarcel J, Krainer AR, Allain $\mathrm{FH}$ : Isolated pseudo-rna-recognition motifs of sr proteins can regulate splicing using a noncanonical mode of rna recognition. Proc Natl Acad Sci U S A (2013) 110(30):E2802-2811.

15. Clery A, Jayne S, Benderska N, Dominguez C, Stamm S, Allain FH: Molecular basis of purine-rich rna recognition by the human sr-like protein tra2-beta1. Nat Struct Mol Biol (2011) 18(4):443450.

16. Berg OG, Winter RB, von Hippel PH: Diffusion-driven mechanisms of protein translocation on nucleic acids. 1. Models and theory. Biochemistry (1981) 20(24):6929-6948.

17. von Hippel PH, Berg OG: On the specificity of DNA-protein interactions. Proc Natl Acad Sci U S A (1986) 83(6):1608-1612.

18. Larouche K, Bergeron MJ, Leclerc S, Guerin SL: Optimization of competitor poly(di-dc).Poly(didc) levels is advised in DNA-protein interaction studies involving enriched nuclear proteins. Biotechniques (1996) 20(3):439-444.

19. O'Brien R, DeDecker B, Fleming KG, Sigler PB, Ladbury JE: The effects of salt on the tata binding protein-DNA interaction from a hyperthermophilic archaeon. J Mol Biol (1998) 279(1):117-125.

20. Spuhler PS, Knezevic J, Yalcin A, Bao Q, Pringsheim E, Droge P, Rant U, Unlu MS: Platform for in situ real-time measurement of protein-induced conformational changes of DNA. Proc NatI Acad Sci U S A (2010) 107(4):1397-1401.

21. Renaud JP, Chung CW, Danielson UH, Egner U, Hennig M, Hubbard RE, Nar H: Biophysics in drug discovery: Impact, challenges and opportunities. Nat Rev Drug Discov (2016) 15(10):679-698.

22. Rant U, Arinaga K, Fujita S, Yokoyama N, Abstreiter G, Tornow M: Electrical manipulation of oligonucleotides grafted to charged surfaces. Org Biomol Chem (2006) 4(18):3448-3455. 

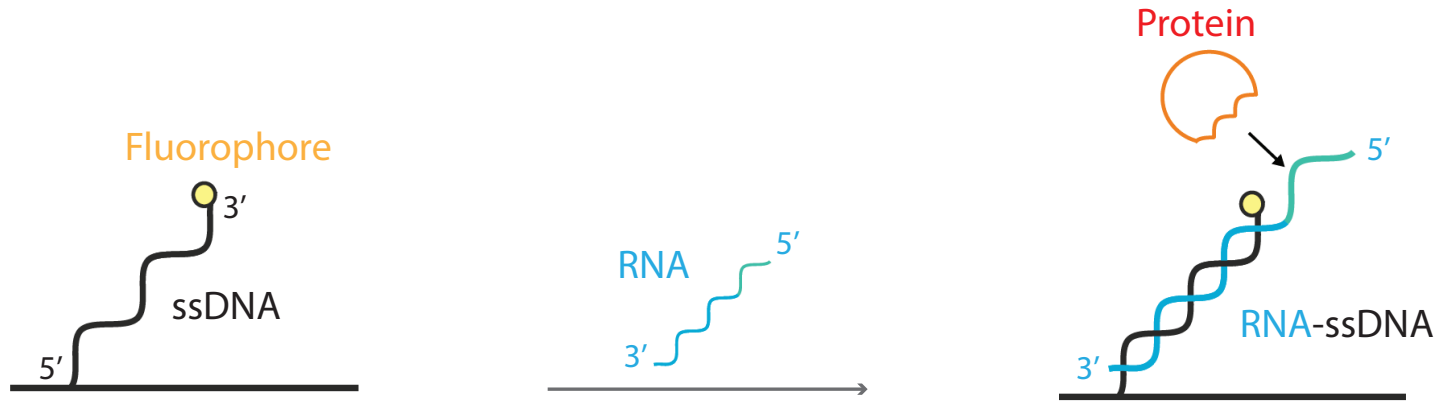

SSDNA: 5'- TAG TCG TAA GCT GAT ATG GCT GAT TAG TCG GAA GCA TCG AAC GCT GAT - 3'

RNA: $3^{\prime}-$ AUC AGC AUU CGA CUA UAC CGA CUA AUC AGC CUU CGU AGC UUG CGA CUA UUUU NNNNNN GAG -5'

Sequence complementary to the ssDNA

Spacer RBP RNA Transcription

target start

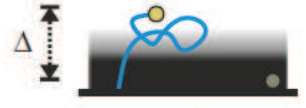

$+/-$

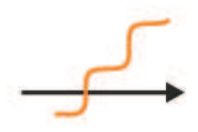

electric field strength

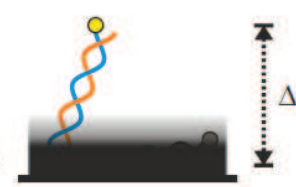

$+/-$
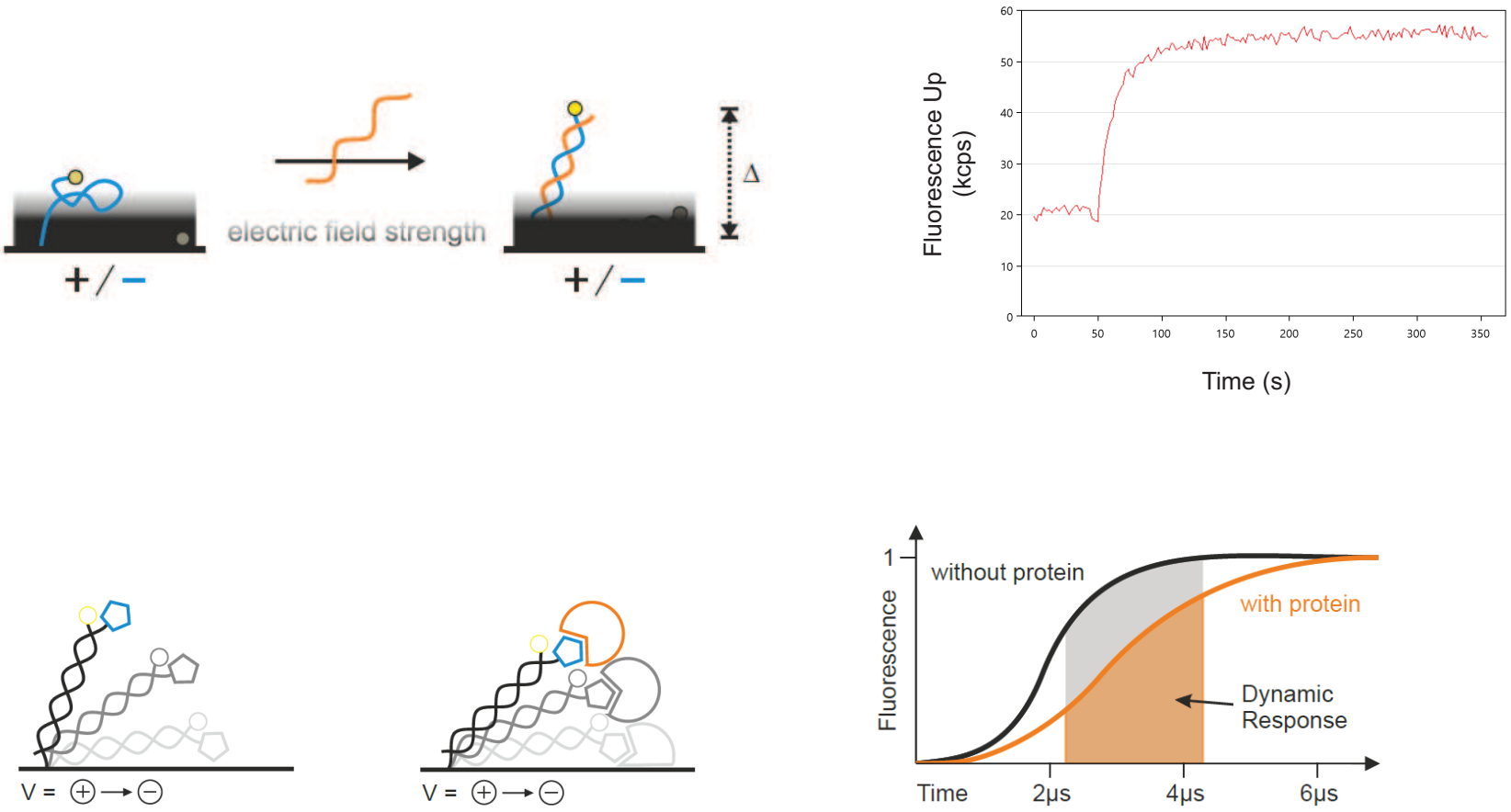

Binding kinetics measurement $\rightarrow \mathrm{k}_{\text {on' }}, \mathrm{k}_{\text {off }}, \mathrm{K}_{\mathrm{D}}$

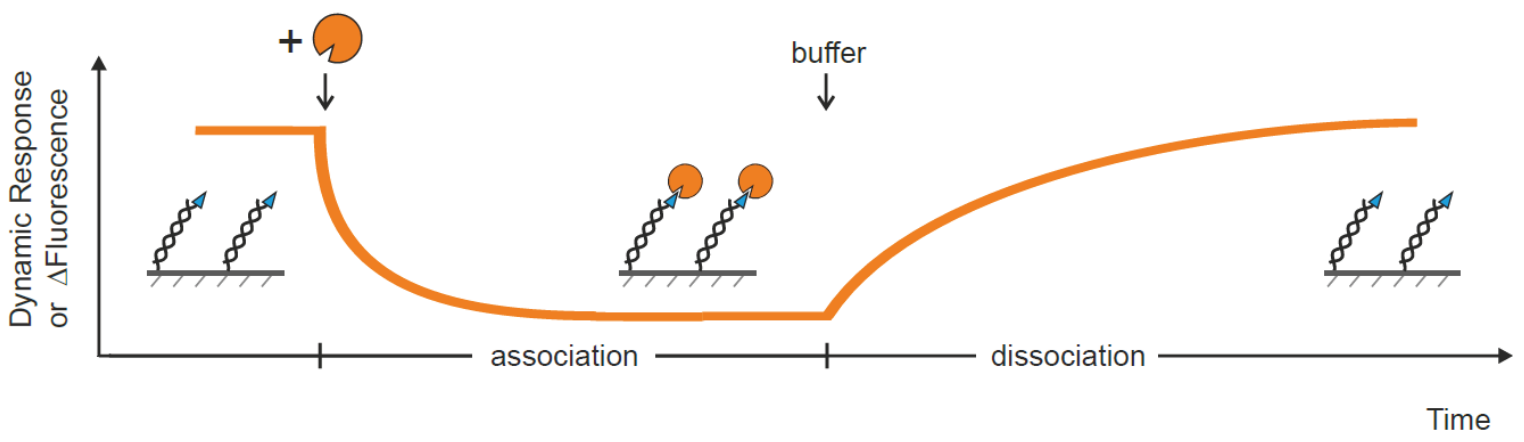

Figure 1 


\section{Fox1 RRM WT + UGCAUGU}
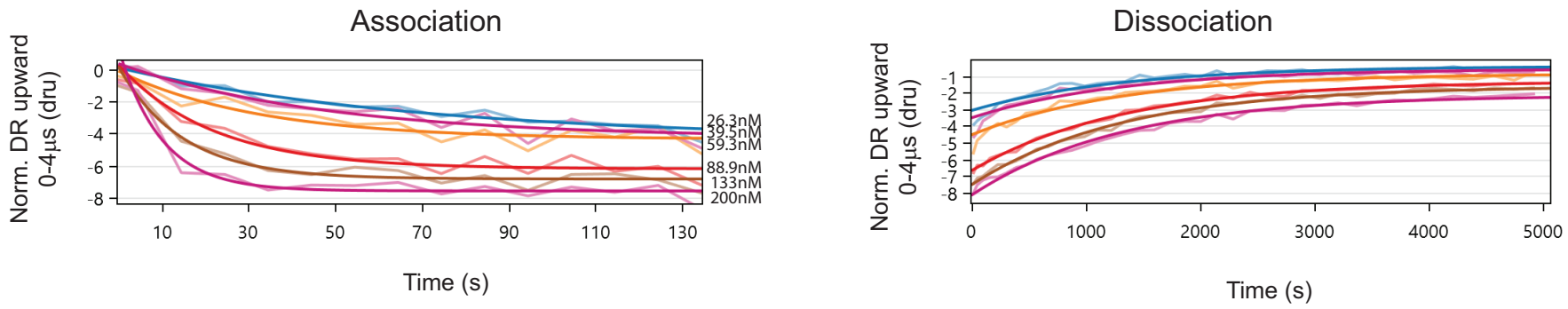

$k_{\text {on }}=5.06+/-0.42 \mathrm{E}+5 \mathrm{M}^{-1} \mathrm{~s}^{-1}$

$k_{\text {off }}=7.55+/-0.14 E-4 s^{-1}$

$K_{D}=1.49+/-0.13 E-9 M(1.49 n M)$

\section{Fox1 RRM WT + polyU}
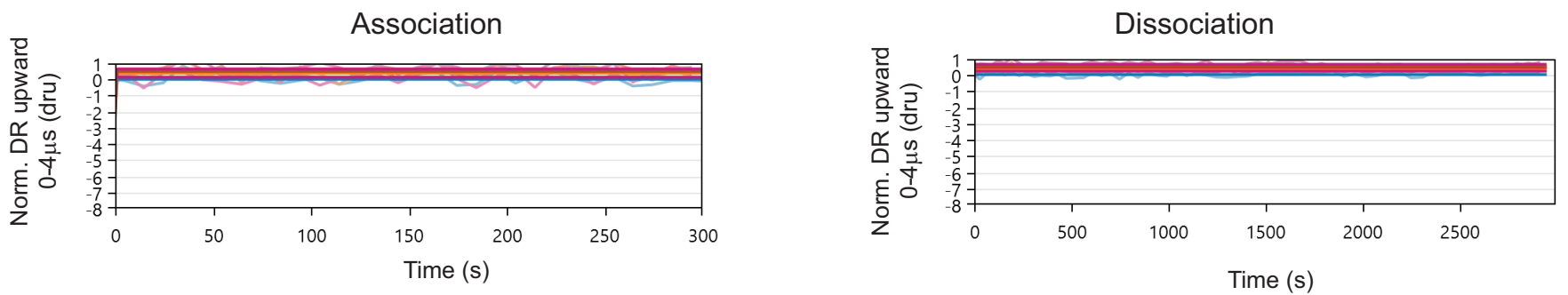

\section{Fox1 RRM F126H + UGCAUGU}
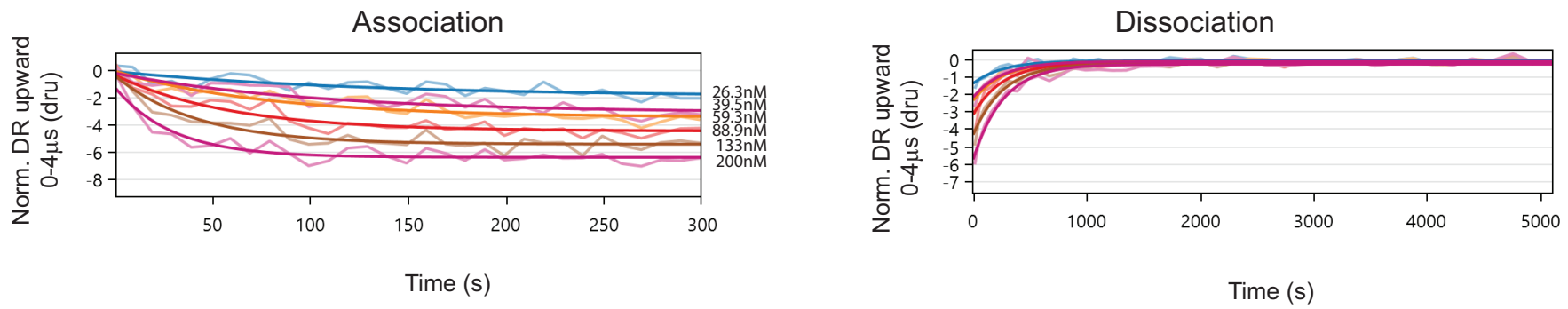

$k_{\text {on }}=1.5+/-0.15 E+5 M^{-1} s^{-1}$

$k_{\text {off }}=3.8+/-0.13 E-3 s^{-1}$

$$
K_{D}=2.53+/-0.27 E-8 M(25.3 n M)
$$

\section{Fox1 RRM S166C + UGCAUGU}

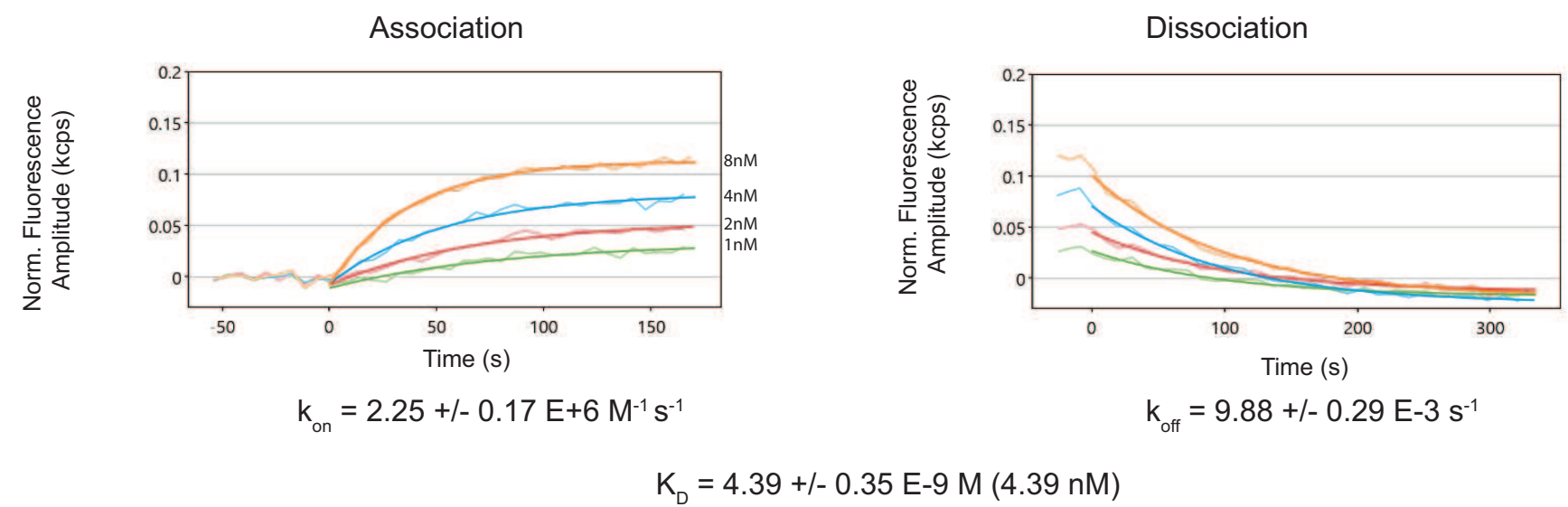

Figure 2 


\section{SRSF1 RRM2 WT + UGAAGGAC}
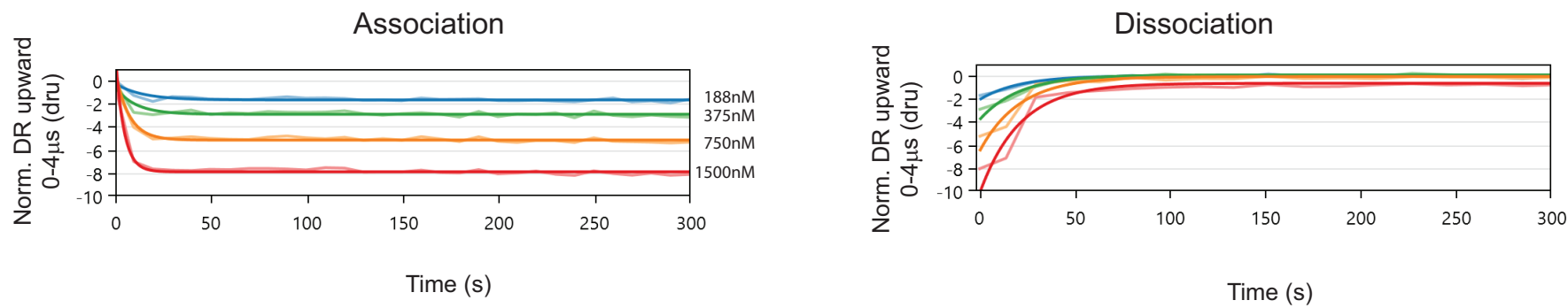

$\mathrm{k}_{\text {on }}=1.24+/-0.09 \mathrm{E}+5 \mathrm{M}^{-1} \mathrm{~s}^{-1}$

$K_{D}=3.86+/-0.28 E-7 M(386 n M)$

SRSF1 RRM2 WT + polyC
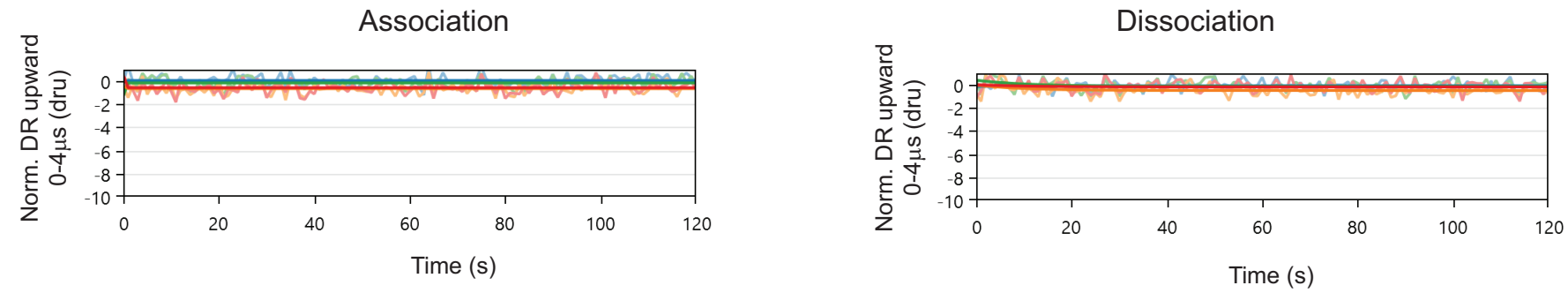

Figure 3 
Tra2- $\beta 1$ RRM WT + AAGAAC
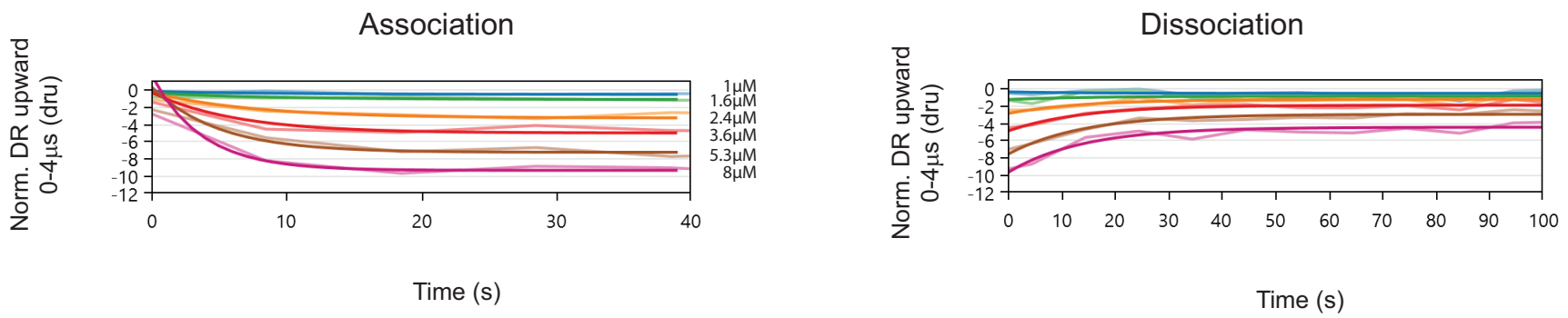

$k_{\text {on }}=2.49+/-0.52 E+4 M^{-1} \mathrm{~s}^{-1}$

$\mathrm{k}_{\text {off }}=7.2+/-0.87 \mathrm{E}-2 \mathrm{~s}^{-1}$

$\mathrm{K}_{\mathrm{D}}=2.89+/-0.7 \mathrm{E}-6 \mathrm{M}(2.89 \mu \mathrm{M})$

Tra2- $\beta 1$ RRM WT + polyU
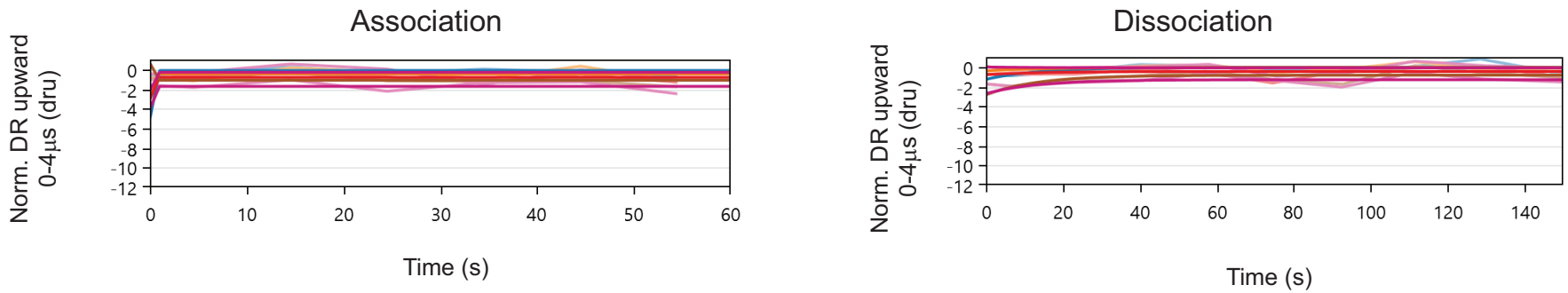

Tra2- $\beta 1$ RRM WT + AAGAAC
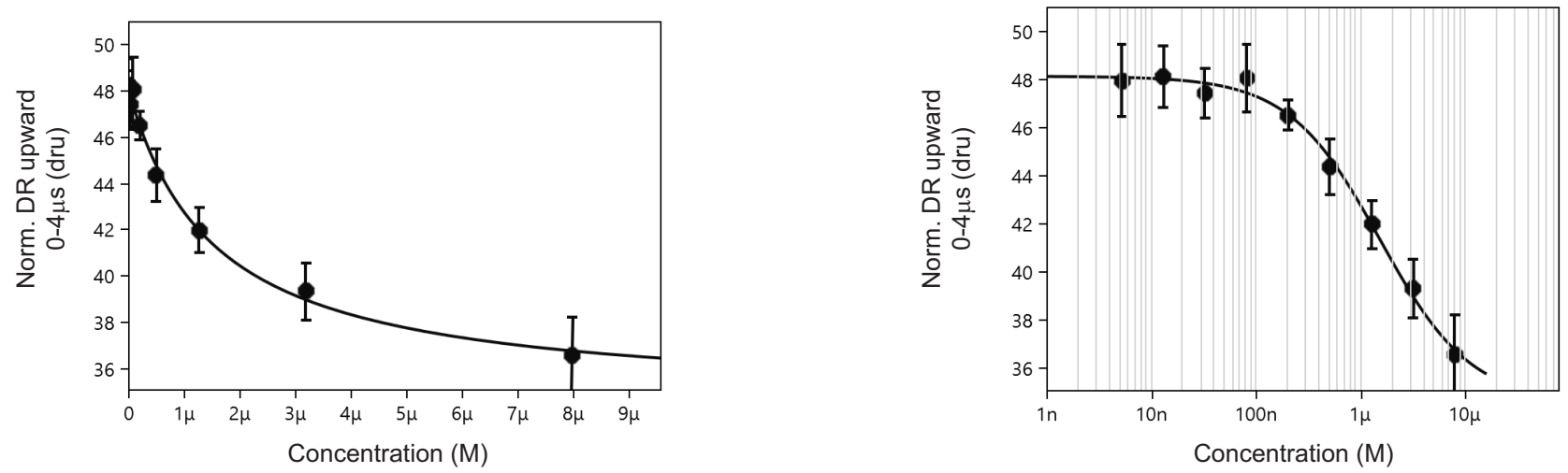

$\mathrm{K}_{\mathrm{D}}=1.53+/-0.14 \mathrm{E}-6 \mathrm{M}(1.53 \mu \mathrm{M})$
$\mathrm{R}^{2}=0.9942$

Figure 4 